\title{
14-3-3 regulates the LNK/JAK2 pathway in mouse hematopoietic stem and progenitor cells
}

\author{
Jing Jiang, ${ }^{1,2}$ Joanna Balcerek, ${ }^{1,2}$ Krasimira Rozenova, ${ }^{1,2}$ Ying Cheng, ${ }^{1,2}$ Alexey Bersenev, ${ }^{1,2}$ \\ Chao Wu, 1,2 Yiwen Song, ${ }^{1,2}$ and Wei Tong',
}

1Division of Hematology, Children's Hospital of Philadelphia, Philadelphia, Pennsylvania, USA. '2Department of Pediatrics, Perelman School of Medicine at the University of Pennsylvania, Philadelphia, Pennsylvania, USA.

\begin{abstract}
Hematopoietic stem and progenitor cell (HSPC) functions are governed by intricate signaling networks. The tyrosine kinase JAK2 plays an essential role in cytokine signaling during hematopoiesis. The adaptor protein LNK is a critical determinant of this process through its inhibitory interaction with JAK2, thereby limiting HSPC self-renewal. LNK deficiency promotes myeloproliferative neoplasm (MPN) development in mice, and LNK loss-of-function mutations are found in human MPNs, emphasizing its pivotal role in normal and malignant HSPCs. Here, we report the identification of 14-3-3 proteins as LNK binding partners. 14-3-3 interfered with the LNK-JAK2 interaction, thereby alleviating LNK inhibition of JAK2 signaling and cell proliferation. Binding of 14-3-3 required 2 previously unappreciated serine phosphorylation sites in LNK, and we found that their phosphorylation is mediated by glycogen synthase kinase 3 and PKA kinases. Mutations of these residues abrogated the interaction and augmented the growth inhibitory function of LNK. Conversely, forced 14-3-3 binding constrained LNK function. Furthermore, interaction with 14-3-3 sequestered LNK in the cytoplasm away from the plasma membrane-proximal JAK2. Importantly, bone marrow transplantation studies revealed an essential role for 14-3-3 in HSPC reconstitution that can be partially mitigated by LNK deficiency. We believe that, together, this work implicates 14-3-3 proteins as novel and positive HSPC regulators by impinging on the LNK/JAK2 pathway.
\end{abstract}

\section{Introduction}

Throughout life, blood cells are continually produced from HSCs that are defined by their multilineage potential and self-renewal capacity. One important signaling axis in hematopoietic stem and progenitor cell (HSPC) expansion and megakaryocyte development is initiated by thrombopoietin (TPO) and its receptor, MPL (1). TPO binding to MPL activates the JAK2 tyrosine kinase, triggering a cascade of signaling events. Downstream signaling molecules include a variety of positive mediators, such as Stats, PI-3K/AKT, and RAS/MAPK (1), together with multiple negative regulators. These negative regulators provide checks and balances at multiple levels to limit cellular responses and prevent oncogenic transformation. The adaptor protein LNK is one important cytokine signaling attenuator.

LNK (also called SH2B3) is a member of an adaptor protein family that does not possess any enzymatic activity. LNK contains several protein-protein interaction domains, including a dimerization domain and proline-rich regions at the amino $(\mathrm{N})$ terminus, a pleckstrin homology $(\mathrm{PH})$ domain in the center, and Src homology 2 (SH2) domain near the carboxyl (C) terminus (2). Each of these domains is important for the inhibitory role of LNK in cytokine-mediated hematopoiesis (3-6). $\mathrm{Lnk}^{-/-}$mice show profound perturbations in hematopoiesis, exhibiting a 3- to 5-fold elevation in white blood cell and platelet counts (6) and increased megakaryocyte numbers in the BM and spleen $(4,6)$. In addition, LNK deficiency also leads to a 10- to 15 -fold

Authorship note: Jing Jiang and Joanna Balcerek contributed equally to this work. Conflict of interest: The authors have declared that no conflict of interest exists. Citation for this article: J Clin Invest. 2012;122(6):2079-2091. doi:10.1172/JCI59719. increase in HSC number and superior multilineage repopulation after BM transplantation (BMT) (7-9).

We and others previously demonstrated that LNK function is partially mediated through TPO/MPL $(4,7,8,10)$. LNK negatively regulates TPO-mediated signaling and megakaryocyte development (4). Moreover, we showed that LNK interacts with phosphorylated JAK2 in a TPO-dependent manner and pinpointed the interaction to the LNK SH2 domain and JAK2 pY813 (8). Importantly, Lnk $^{-1}$ HSPCs display potentiated JAK2 activation in response to TPO, suggesting that LNK controls HSC self-renewal in part through the MPL/JAK2 pathway (8). However, the mechanisms by which LNK attenuates JAK2 activity are poorly understood.

The amplitude and duration of cytokine receptor signaling is tightly controlled, and aberrant regulation predisposes HSPCs to myeloproliferative neoplasms (MPNs) (11). An activating mutation (V617F) in JAK2 is found at high frequencies in MPNs (11). Consistent with a role of LNK in constraining JAK2-regulated cell growth, loss of LNK accelerates JAK2(V617F)-induced MPNs in mice (12). LNK deficiency enhanced cytokine-independent JAK/ Stat signaling and augmented the ability of oncogenic JAK2 to expand myeloid progenitors in vitro and in vivo (12-14). The relevance of these findings to human disease is underscored by the recent identification of LNK loss-of-function mutations in human patients with MPN (15-17).

In search of a mechanism for LNK function, we describe here the identification of 14-3-3 proteins as the robust LNK binding partners. $14-3-3$ proteins are abundant 28 - to $33-\mathrm{kDa}$ acidic polypeptides found in all eukaryotic organisms (18) that play important roles in a wide range of biologic processes, including cell cycle regulation, signaling transduction, metabolism control, apopto- 


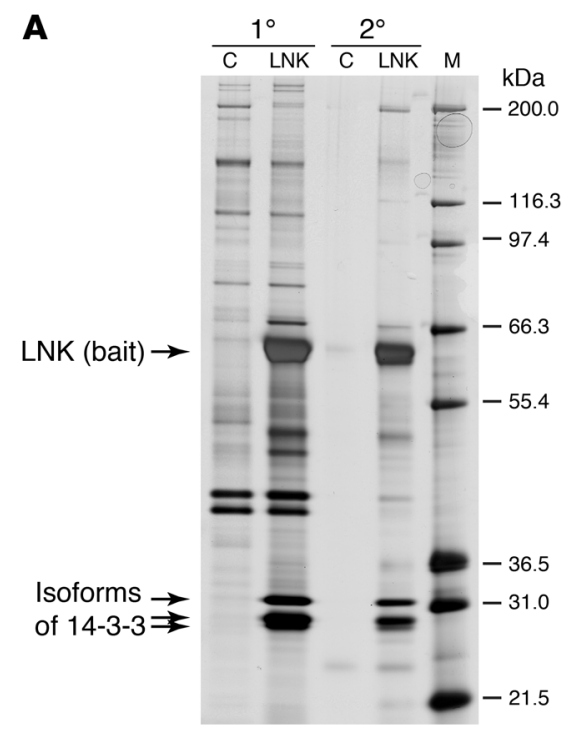

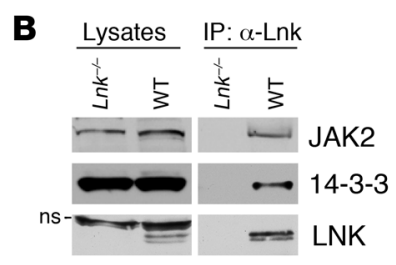

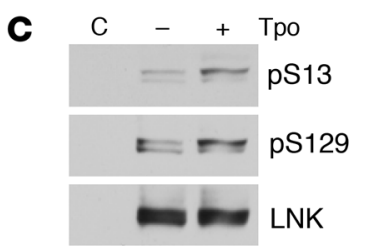

\section{Figure 1}

Identification of 14-3-3 proteins as LNK binding partners. (A) Cytoplasmic fractions of 32D-B/A parental cells (C) or cells stably expressing Flag/HA-LNK (LNK) were isolated and precipitated with anti-Flag $\left(1^{\circ}\right)$ and anti-HA antibodies $\left(2^{\circ}\right)$ sequentially. IP eluates were subjected to SDSPAGE and visualized by silver staining. M, marker. (B) LNK interacts with endogenous 14-3-3. Protein lysates from WT and $L n k^{-1-}$ spleens were either directly subjected to WB analysis or IP with anti-LNK antibodies, followed by WB analysis with indicated antibodies. ns, nonspecific. (C) 32D-B/A cells stably expressing Lnk were stimulated with or without Tpo for 10 minutes. Cell lysates were subjected to WB analysis with phospho-specific antibodies to pS13 and pS129 sites in LNK or total LNK protein. sis, and control of gene transcription (19). 14-3-3 proteins are highly conserved, and 7 family members are found in mammals $-\beta, \gamma, \varepsilon, \sigma, \tau$ (also referred to as $\theta$ ), $\zeta$, and $\eta$. 14-3-3 binding by client proteins requires serine/threonine phosphorylation within 1 out of 2 known consensus peptide motifs, RSXPSXP (mode 1) and $\mathrm{RX}(\mathrm{Y} / \mathrm{F}) \mathrm{XpSXP}$ (mode 2 ), where $\mathrm{pS}$ represents phospho-serine (20-22). 14-3-3 proteins can form homodimers and heterodimers, which contribute to their ability to modulate the function of their binding partners (18).

Although 14-3-3 proteins participate in diverse cellular functions (23), their roles in blood development have remained largely undefined. Mice lacking the $\varepsilon$, $\sigma$, or $\tau$ isoforms display a perinatal lethality due to defects in neurons, keratinocytes, and cardiomyocytes, respectively (24-26), precluding further studies of their hematopoietic functions in adult mice. In contrast, mice lacking the $\gamma$ or $\xi$ isoforms appear grossly normal $(27,28)$. Mice deficient for 14-3-3 $\beta$ or $\eta$ have not been reported. Recent studies showed that disrupting 14-3-3/ligand association in transformed hematopoietic cell lines induced apoptosis in part by disrupting the interaction between 14-3-3 and FOXO3a, implicating 14-3-3 as a potential therapeutic target in leukemia (29). Two recent reports point to a role of 14-3-3 proteins in stress erythropoiesis $(30,31)$. However, the function of 14-3-3 proteins in steady-state hematopoiesis and HSPC expansion has not been explored.

Here, we showed that LNK is phosphorylated at 2 serine residues that serve as 14-3-3 binding sites. Importantly, 14-3-3 abrogated the LNK-JAK2 interaction, thereby alleviating LNK inhibitory function in both JAK2 signaling and cell growth. Furthermore, 14-3-3 binding was necessary and sufficient to maintain LNK in an inactive state by sequestering it from its target JAK2 in cell lines. An essential role of the LNK-14-3-3 interaction in HSPC development in vivo was revealed by BMT experiments in which 14-3-3-depleted HSPCs failed to normally reconstitute hematopoietic development. 14-3-3 functions in part via LNK, since the deleterious effects of 14-3-3 knockdown were mitigated in the absence of LNK. Consistent with our model, STAT5 activation is impaired in 14-3-3depleted HSPCs. In concert, our work uncovered LNK as a dynamic adaptor protein controlled by a phosphorylation-dependent interaction with 14-3-3. The highly defined and signal-dependent inter- action between LNK and partner proteins elevates its potential as a therapeutic target for stem cell therapies and MPNs.

\section{Results}

Phosphorylation-dependent interaction between LNK and 14-3-3 proteins. Our previous studies showed that LNK constrains both normal and malignant HSPC expansion in part through limiting JAK2 activity (8). However, the underlying mechanisms are poorly understood. We therefore set out to explore LNK regulatory mechanisms by identifying LNK interacting complexes that we believe to be novel.

To overcome the growth inhibitory effect of LNK, 32D cells were engineered to coexpress BCR-ABL (hereafter referred to as 32D-B/A cells) $(8,12)$. Thus, we were able to generate $32 \mathrm{D}$ cell lines stably expressing Flag-HA double-tagged LNK. Cytoplasmic protein extracts were subjected to anti-Flag immunoprecipitation (IP), followed by Flag peptide elution and anti-HA IP. Bound material was eluted with glycine and fractionated by SDS-PAGE (Figure 1A) and the resulting gel slices were excised for protein identification by mass spectrometry. In addition to the known LNK partner JAK2, 14-3-3 proteins $\beta, \gamma, \varepsilon, \zeta, \eta$, and $\tau$ were enriched in our purification (Figure 1A and Supplemental Figure 1; supplemental material available online with this article; doi:10.1172/JCI59719DS1).

The interaction between endogenous LNK and 14-3-3 was validated in cells from WT and $L n k^{-/-}$spleens, with JAK2 serving as positive control (Figure 1B). Subsequently, we mapped the interaction between LNK and 14-3-3 \& (14-3-3) in 293T cells. The Scansite (32) analysis of mouse LNK protein predicted 2 high-stringency 14-3-3 binding motifs - S13 and S129 - near the N terminus. These 2 serines were converted singly (S13A or S129A) or doubly (2SA) into alanines, and their effects were examined by co-IP of Flag-LNK with myc-14-3-3 or endogenous 14-3-3 in 293T cells (Figure 2A). Although, WT LNK readily associated with both forms of 14-3-3, the single serine mutants were impaired in 14-3-3 binding, especially the S129A version. The 2SA mutations completely abolished the LNK-14-3-3 interaction (Figure 2A). The reverse IP with anti-myc antibodies showed similar results (Figure 2A). Moreover, we confirmed these results in 32D-B/A cells stably expressing WT LNK or the LNK mutants (Figure 2, B and C). The N termi- 

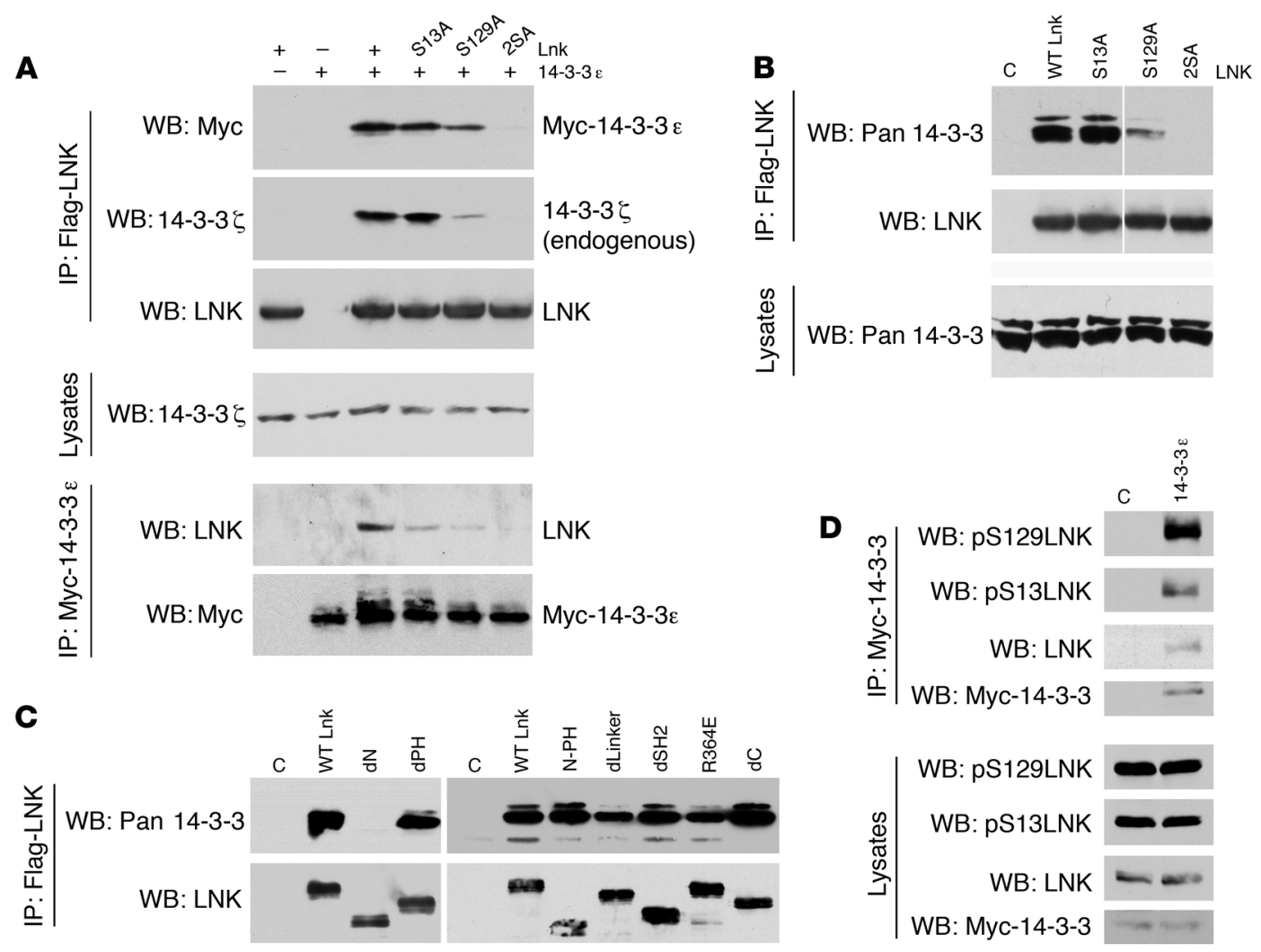

Figure 2

14-3-3 binds to LNK at the S13 and S129 sites. (A) 293T cells were cotransfected with myc-14-3-3 and Flag-Lnk or Lnk mutants. Lysates were precipitated with anti-Flag (top) or anti-myc antibodies (bottom), followed by WB using indicated antibodies. (B) Endogenous 14-3-3 binds to LNK at the S13 and S129 sites. Lysates from 32D-B/A cells expressing Flag-LNK or LNK mutants were precipitated with anti-Flag antibodies, followed by WB using anti-pan-14-3-3 and -LNK antibodies. (C) LNK associates with endogenous 14-3-3 through its $\mathrm{N}$ terminus where the S13/S129 sites are located. Experiments were performed similarly to those in B. d, deletion; N, N terminus; C, C terminus; Linker, the region between the PH and $\mathrm{SH} 2$ domain. All the lanes were run on the same gel but were noncontiguous. (D) 14-3-3 pulls down serine phosphorylated LNK. 32D-B/A cells expressing LNK and myc-14-3-3 were established. Lysates were precipitated with anti-myc antibodies, followed by WB using antibodies to $\mathrm{pS} 13$, pS129, or total LNK.

nus of LNK comprising S13 and S129 was required for the 14-3-3 interaction, whereas the $\mathrm{PH}$ or $\mathrm{SH} 2$ domains were dispensable for 14-3-3 binding (Figure 2, B and C). The involvement of consensus serine motifs in the 14-3-3 interaction suggests that phosphorylation of LNK at the S13 and S129 sites regulates 14-3-3 binding.

To monitor the phosphorylation state of LNK, we generated phospho-specific antibodies to pS13 and pS129. Using these reagents in 32D cells, we found that 14-3-3 associates with LNK in a manner dependent on pS13 and pS129 phosphorylation (Figure 2D). Furthermore, the phosphorylation of these 2 sites is moderately increased upon TPO stimulation in 32D cells stably expressing MPL and Flag-LNK, although there was a notable basal level of phosphorylation (Figure 1C). Importantly, LNK purified from 32D-B/A cells was subjected to mass spectrometric analysis, which confirmed that both S13 and S129 are indeed phosphorylated in vivo (data not shown). Together, these results reveal 14-3-3 proteins as components of the LNK complex and suggest that signal-dependent phosphorylation modulates LNK activity via 14-3-3 association.

14-3-3 restrains growth inbibition by $L N K$. To determine the functional consequences of the LNK-14-3-3 interaction, we compared the growth inhibitory function of WT LNK and 14-3-3 binding- defective LNK in 32D cells. Lnk cDNAs were introduced into a bicistronic $\mathrm{POZ}$ retroviral vector that coexpresses the human IL-2 receptor (IL-2R). After infection of $32 \mathrm{D}$ cells, the fraction of IL- $2 \mathrm{R}^{+}$ cells was monitored by flow cytometry and compared to that of cells infected with control vector (Figure 3A). WT LNK expression impaired cell growth as previously reported $(3,4)$. However, LNK 2SA double mutants and the S129A single mutants conferred an even more pronounced growth disadvantage (Figure $3 \mathrm{~A}$ ). To confirm the growth inhibitory effects of LNK, cell numbers of infected cells were measured daily. LNK-expressing 32D cells showed blunted cell growth, while the vector control cells exhibited exponential growth. Importantly, cells expressing LNK 2SA and S129A showed a markedly slower growth rate than cells expressing LNK WT (Figure $3 \mathrm{~B}$ ). Therefore, the ability of LNK to associate with 14-3-3 inversely correlates with its growth inhibitory activity, suggesting that 14-3-3 constrains the activity of LNK.

To examine whether LNK functions similarly in primary hematopoietic progenitor cells, we infected lineage $\left(\mathrm{Lin}^{-}\right)$progenitors from $L n k^{-/-}$BM with retrovirus encoding WT or mutant Lnk and determined the cell cycle profile by measuring BrdU incorporation. WT LNK substantially impaired cell cycle progression, as reflected 

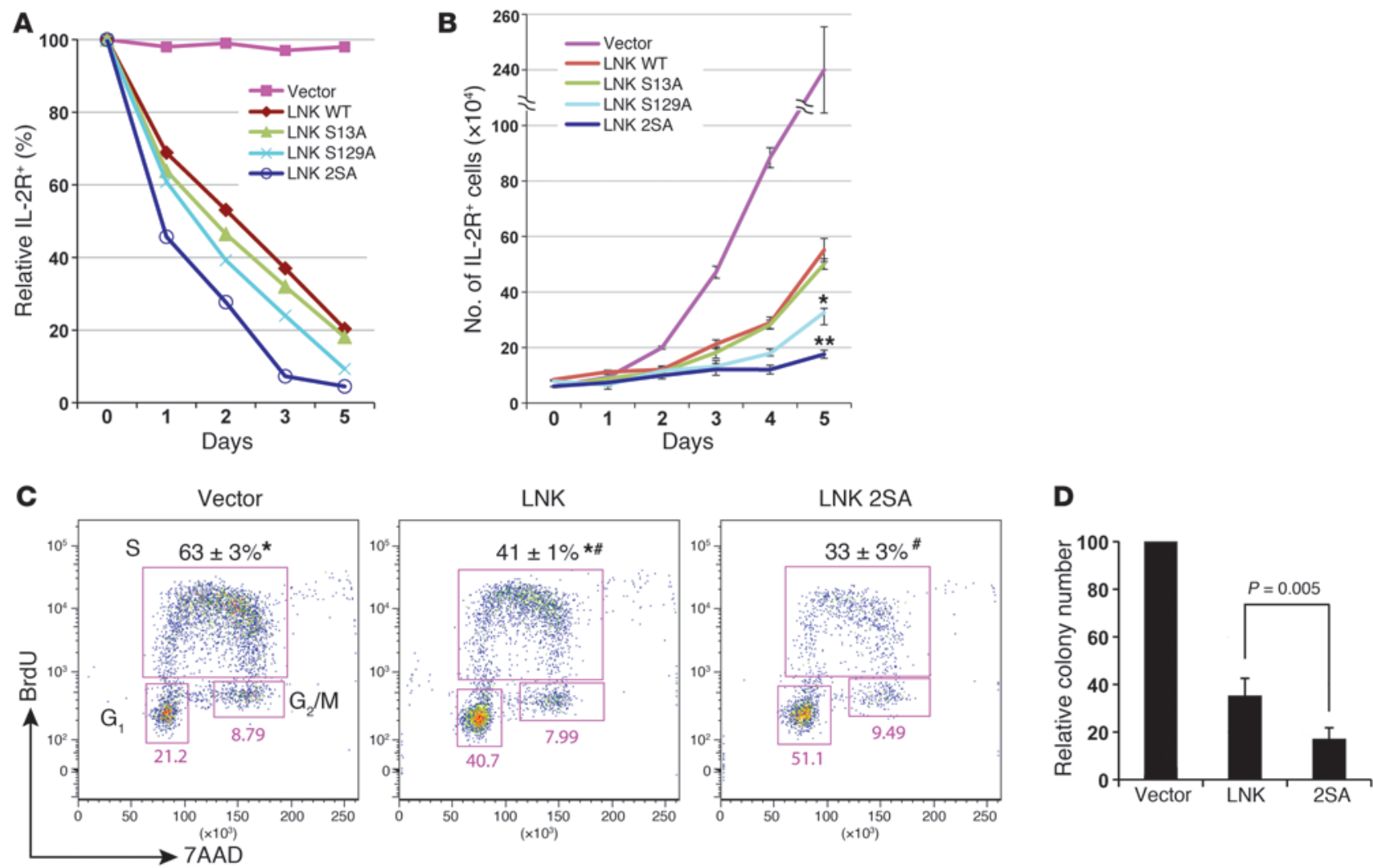

\section{Figure 3}

14-3-3 binding restrains LNK growth inhibitory function. (A) 32D cells were infected with retroviruses encoding either WT or mutant Lnk in a bicistronic vector, pOZ, with IL-2R as a marker. We determined the proportion of infected cells as the proportion of those that expressed IL-2R 2 days after infection, and set that day as day 0 . We then measured the IL-2R+ fraction every day afterward, and the percentage of IL-2R+ cells relative to the initial infection rate is plotted. Representatives of 3 independent experiments are shown. (B) 32D cells were infected with pOZ-Lnk, as described in A. The number of cells with IL-2R+-infected populations was enumerated daily, calculated, and plotted (mean \pm SD). ${ }^{\star} P=0.003,{ }^{\star \star} P=0.0001$, when compared with LNK WT. (C) Primary Lin- BM cells from $\mathrm{Lnk}^{-1-}$ mice were infected with retroviruses encoding either vector alone or pOZ-WT or mutant Lnk and cultured in vitro in a cocktail of cytokines. BrdU was added the day after infection. Cell cycle profiles for BrdU and 7'-AAD were analyzed on IL-2R+-infected cells $(n=3)$. Numbers represent the percentages of BrdU' cells in the S-phase of the cell cycle. ${ }^{*} P=0.0003,{ }^{\#} P=0.007$. (D) Infected Lin- BM cells were plated in methylcellulose culture (M3434, Stem Cell Technologies), and the colony numbers are shown $(n=3) . P=0.005$, Lnk versus $2 \mathrm{SA}$. $(\mathbf{A}, \mathbf{B}$, and $\mathbf{D})$ Average \pm SD.

in the reduced fraction of cells in $S$ phase and increased population of cells in the $\mathrm{G}_{1}$ phase of the cell cycle (Figure 3C). These growth inhibitory effects were even more pronounced when LNK 2SA was expressed (Figure 3C). Changes in the rate of apoptosis do not account for the observed effects (Supplemental Figure 2). Furthermore, when plated in methylcellulose cultures, LNKexpressing Lin- BM cells produced markedly reduced colony numbers when compared with those of the control (Figure 3D). The inhibitory effects on colony formation were further augmented by LNK 2SA (Figure 3D). Taken together these results indicate that 14-3-3 restrains inhibitory function of LNK in cell proliferation.

14-3-3 interferes with LNK's inhibition of JAK2 signaling. Since LNK slows cell growth by inhibiting JAK2, it is plausible that 14-3-3 antagonizes effects of LNK on JAK2. Therefore, we measured the effects of WT and mutant LNK on cytokine-stimulated JAK2 activity and its downstream signal transducers. LNK and LNK 2SA were expressed in 32D cells stably expressing MPL (32D/MPL cells) using the $\mathrm{POZ}$ retroviral vector. After TPO stimulation, we measured the activities of JAK2 and key downstream effectors
(STAT5, MAPK, and AKT) by flow cytometry with phospho-specific antibodies $(3,4,8)$. LNK 2 SA triggered a more pronounced inhibition in JAK2 activity as well as that of its signal transducers when compared with that of WT LNK (Figure 4). Thus, our data indicate that 14-3-3 binding impairs the ability of LNK to inhibit the JAK2 signaling pathway.

14-3-3 impairs the LNK-JAK2 interaction. To study the mechanism by which 14-3-3 inhibits LNK function, we investigated whether 14-3-3 interferes with the LNK-JAK2 interaction (8). Myc-tagged forms of JAK2 and 14-3-3 were coexpressed with Flag-WT LNK or LNK 2SA in $293 \mathrm{~T}$ cells, and their association was assessed by co-IP (Figure 5A). As expected, WT LNK associated with JAK2 and 14-3-3 as well as endogenous 14-3-3 $\zeta$ (Figure 5A). Importantly, LNK 2SA displayed increased myc-JAK2 binding accompanied by a reciprocal decrease in 14-3-3 association (Figure 5A). Thus, our data suggest that 14-3-3 binding inhibits LNK-JAK2 interaction. These results suggest that the physical disruption of the LNKJAK2 interaction accounts for at least some of the growth promoting effects of 14-3-3 in hematopoietic cells. 

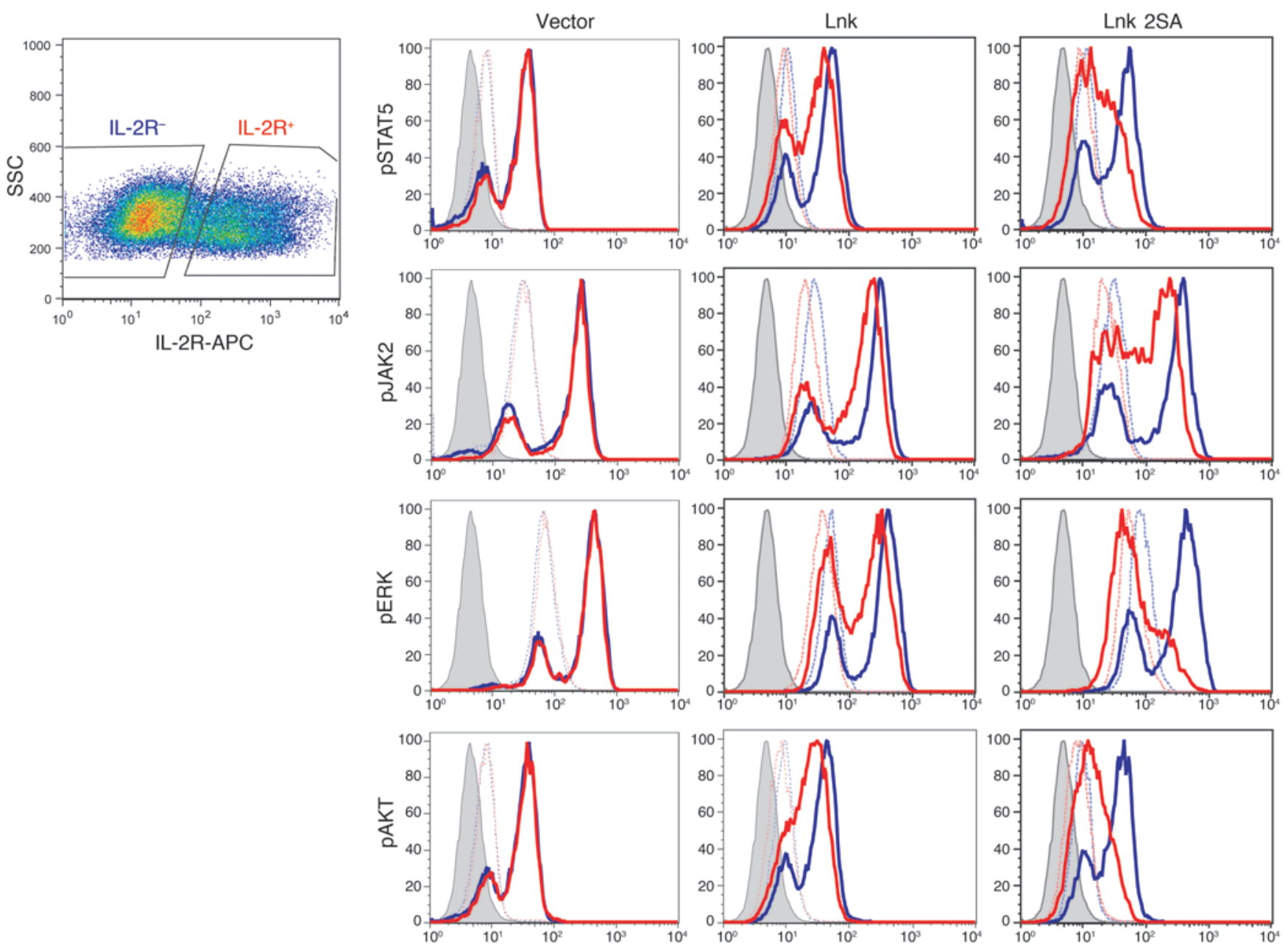

Figure 4

14-3-3 binding attenuates LNK inhibition of cytokine-induced JAK2 activation and downstream signaling. 32D/MPL cells were infected with pOZ retroviruses encoding either Lnk WT or Lnk 2SA. Cells were either left unstimulated or stimulated in $10 \mathrm{ng} / \mathrm{ml}$ TPO for $10 \mathrm{minutes}$. Cells were analyzed for IL-2R expression as well as phosphorylation of JAK2, STAT5, AKT, or ERK, using flow cytometry. Histograms of unstimulated and stimulated cells from the same cell line are overlaid and shown here. The infected IL-2R+ population is labeled in red, while the uninfected IL-2R- population is shown in blue. Dashed lines indicate unstimulated cells, while solid lines indicate stimulated cells. Shaded areas represent secondary antibody-only controls. Representatives of 3 independent experiments are shown.

The inhibition of the LNK-JAK2 interaction by 14-3-3 could occur through direct contacts. Alternatively, 14-3-3 might indirectly influence the LNK-JAK2 interaction via associated factors. To distinguish between these possibilities, we reconstituted FlagLNK, myc-JAK2, and myc-14-3-3 alone or in combination in the baculovirus-sf9 expression system (Figure 5B). Cell lysates were precipitated with either anti-Flag or myc antibodies followed by Coomassie staining of the eluates. WT LNK was found to associate with both JAK2 and 14-3-3 (Figure 5B, lane 1). LNK 2SA failed to bind 14-3-3 but displayed increased association with JAK2 (Figure 5B, lane 2). Importantly, in the absence of 14-3-3, WT LNK and LNK 2SA associated with JAK2 equally (Figure 5B, lane 3 and 4). As controls, noninfected sf9 cells (Figure 5B, lane 5 and 9), LNK or LNK 2SA alone (Figure 5B, lane 6 and 7), and JAK2 or 14-3-3 alone (Figure 5B, lane 10 and 11) are shown. These data suggest that LNK interacts with 14-3-3 directly through S13 and S129 residues.
14-3-3 as a critical regulator of LNK function. The 2SA mutations augment the inhibitory function of LNK while reducing 14-3-3 binding. To rule out the possibility that these mutations impair binding to additional as yet unknown proteins, we specifically restored 14-3-3 binding to LNK 2SA and measured its activity. To this end, we fused a short 14-3-3 binding peptide sequence, referred to as R18, to the N terminus of LNK 2SA. R18 mediates phosphorylation-independent binding to all 14-3-3 isoforms (Figure 6A and refs. 33, 34). A mutant R18 peptide (R18KK), in which the core motif was altered to disrupt 14-3-3 binding (WLLLLE $\rightarrow$ WLLLL $\underline{K}$ ), served as control (34). Co-IP experiments showed that R18 but not R18KK restored 14-3-3 binding to LNK 2SA (Figure 6A). To examine whether loss of 14-3-3 binding was indeed accountable for the increased LNK 2SA activity in hematopoietic cells, different LNK expression constructs were introduced into 32D cells, and cell growth was measured as above. R18-LNK 2SA impaired cellular proliferation to an extent virtually identical to that of WT 


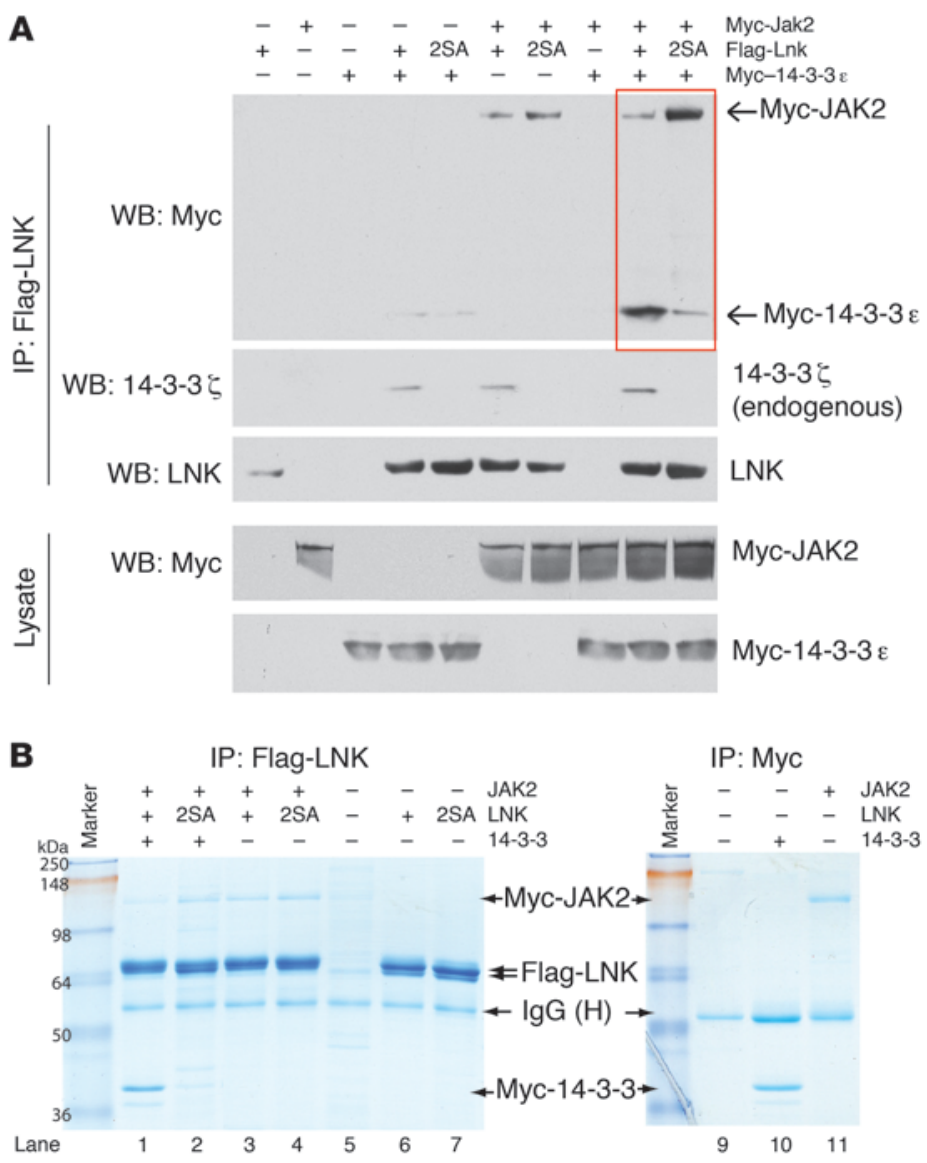

\section{Figure 5}

LNK directly interacts with 14-3-3 and JAK2, and 14-3-3 binding inhibits LNK-JAK2 association. (A) 293T cells were transfected with a series of combinations of constructs as indicated. Cell lysates were precipitated with anti-Flag antibodies and blotted with indicated antibodies. Total cell lysates (pre-IP) were probed with anti-myc antibodies. (B) Recombinant Flag-LNK or 2SA, myc-JAK2, and myc-14-3-3 proteins were reconstituted either separately or together in insect sf9 cells through baculoviral infections. Sf9 lysates were precipitated with anti-Flag or myc antibodies, followed by Coomassie staining of the eluates. Representative results of 2 independent experiments are shown.
LNK (Figure 6B). In contrast, R18KK-LNK 2SA behaved the same as LNK 2SA. We conclude that the specific recruitment of 14-3-3 via S13 and S129 regulates LNK activity.

Phosphorylation of LNK at S13 and S129 by glycogen synthase kinase 3 and $P K A$, respectively. Given that phosphorylation of LNK is critical for 14-3-3 binding, we sought to identify the responsible kinase(s). In silico analysis suggested $\mathrm{S} 13$ as a potential substrate for glycogen synthase kinase 3 (GSK3). To examine GSK3 in vivo, we used 2 GSK3 inhibitors: 6-bromoindirubin-3'-oxime (6BIO) or CHIR99021 (CHIR) (35). Both compounds reduced LNK S13 phosphorylation, confirming GSK3 as a relevant LNK kinase in intact cells (Figure 7A). We next examined the effects of GSK3 inhibition on 14-3-3 binding. Co-IP experiments revealed that $6 \mathrm{BIO}$ or CHIR interfered with the LNK-14-3-3 interaction (Figure $7 \mathrm{~A})$. These results suggest that GSK3 impinges on LNK function by promoting 14-3-3 binding.

S129 of LNK resides within a consensus PKA target phosphorylation site. Hence we examined all PKA isoforms in an in vitro kinase assay using LNK peptides as substrates. All forms of PKA examined, but not those PKC, were able to directly phosphorylate S129 (Figure 7B). In addition, the PKA inhibitor H-89 reduced LNK S129 phosphorylation and 14-3-3 binding in cells (Figure 7C). To extend these studies in an unbiased fashion, we also carried out a comprehensive in vitro kinase screen with 36 candidate serine/threonine kinases. ROCK, Aurora, p90 ribosomal S6 kinases showed in vitro activities toward S129. However, their pharmacologic inhibition in vivo did not produce any measurable changes in S129 phosphorylation or 14-3-3 binding (data not shown). Together, these results implicate PKA as the relevant LNK S129 kinase.

To examine whether JAK2 signaling regulates the LNK-14-3-3 interaction, we performed co-IP experiments in the presence of active (V617F) and kinase-inactive (Y1007/1008F) versions of JAK2 or a constitutively active form of MPL (W5151K). None of these conditions affected the LNK-14-3-3 interaction (Supplemental Figures 3 and 4), consistent with a model in which serine phosphorylation of LNK acts upstream to regulate LNK function, while JAK/STAT signaling is a downstream effector of LNK. Future work will establish the signals that stimulate PKA and GSK3.

14-3-3 sequesters LNK in the cytoplasm from plasma membrane-proximal JAK2. To elucidate mechanisms by which 14-3-3 interferes with the LNK-JAK2 interaction, we assessed the cellular localization of LNK and JAK2 using confocal microscopy. 293T cells were transfected with pEGFP-Jak2, pOZ-Lnk (Flag/HA-Lnk), or Lnk 2SA either singly or in combination. Cells were stained with antiGFP and anti-HA antibodies, respectively. Confocal microscopy images showed that the majority of WT LNK was cytoplasmic, with modest staining at the plasma membrane (Figure 8), in agreement with previous reports (36). In contrast, a much larger fraction of LNK 2SA was found at the plasma membrane (Figure 8). Notably, we observed marked colocalization of LNK 2SA and membrane-bound JAK2. These data suggest that 14-3-3 sequesters LNK in the cytoplasm to limit its association with plasma membrane-proximal JAK2.

HSPC reconstitution in mice requires 14-3-3 proteins. Our results suggest a previously unknown function of $14-3-3$ proteins in regu- 
A
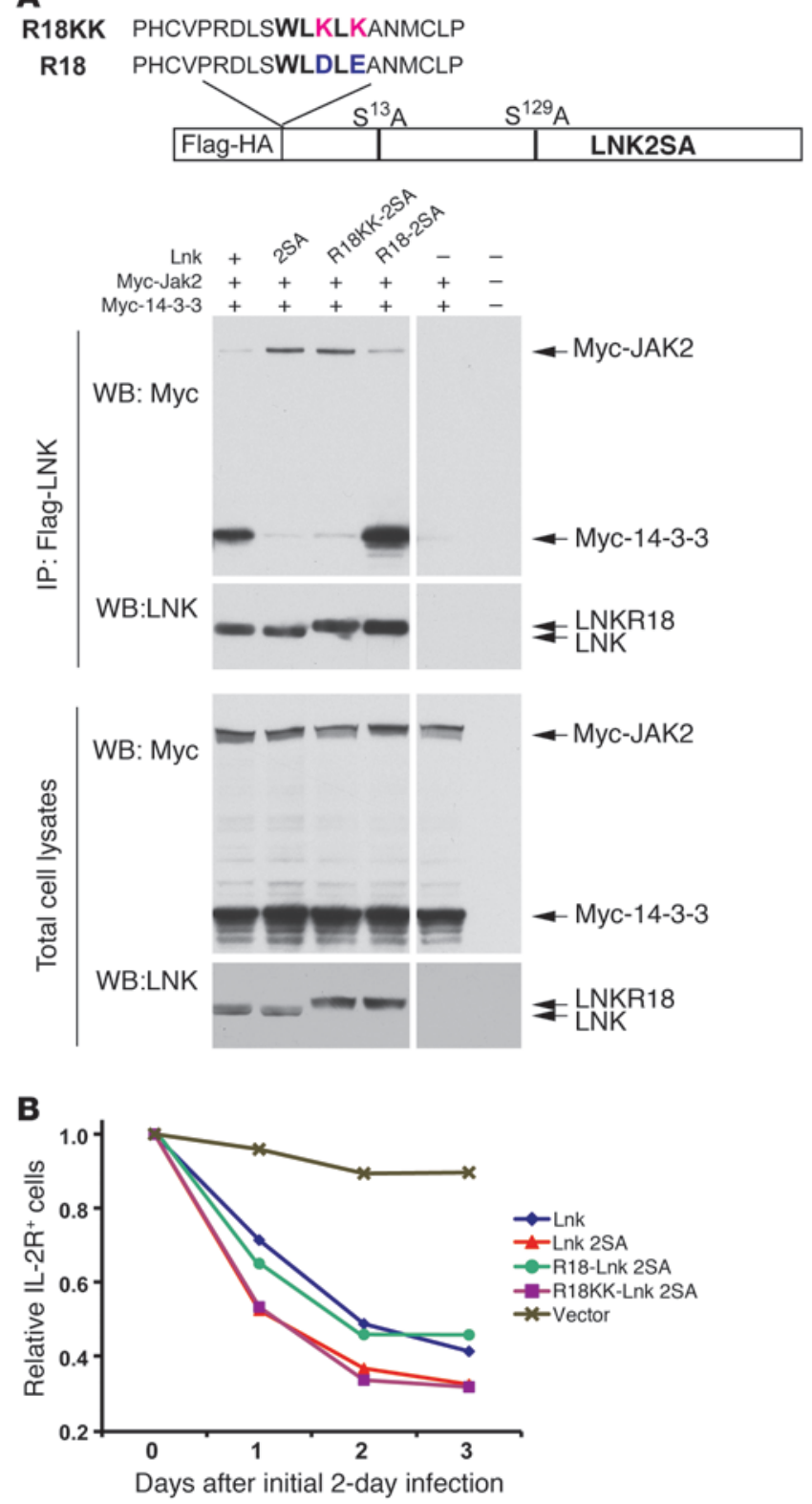

lating HSPC signaling via LNK and JAK2. To assess the role of 14-3-3 in HSPCs in vivo, we set out to deplete 14-3-3 proteins using shRNA-mediated knockdown followed by BMT.

Four 14-3-3 isoforms $(\beta, \varepsilon, \zeta$, and $\eta$ ) were previously found in the BM at the mRNA level $(37,38)$, with 14-3-3 $\eta$ being undetectable at the protein level (data not shown). We therefore dissected the role of individual 14-3-3 isoforms in HSPCs by knocking down individual 14-3-3 isoforms (i.e., $\beta, \varepsilon$, and $\xi$ ) then performing BMT (Figure 9, $A$ and $B)$. Lin $^{-}$BM cells were infected with pLKO lentiviruses expressing shRNAs targeting 14-3-3 proteins or, as control, luciferase (Luc). Western blotting (WB) analysis identified shRNA clones that effectively diminished the production of individual $\beta$, $\varepsilon$, and $\zeta$ isoforms (Figure 9A). Infected Lin $^{-}$BM cells were transplanted into lethally irradiated recipient mice. FACS analysis measuring the percentage of $\mathrm{GFP}^{+}$cells was carried out prior to transplantation as well as monthly thereafter (Figure 9B). We found that

\section{Figure 6}

14-3-3 is sufficient to restrain LNK binding to JAK2 and growth inhibition. (A) The top panel shows the sequences of synthetic 14-3-3 binding peptides $\mathrm{R} 18$ and R18KK. The core motif WLKLK is indicated in bold. The blue letters indicate Wildtype R18 motif sequence, while the red indicates the mutated residues The bottom panel shows biochemical studies using various LNK constructs. 293T cells were transfected with different combinations of constructs, consisting of myc-Jak2, myc-14-3-3 $\varepsilon$, and Flag-Lnk or Lnk mutants. Cell lysates were precipitated with anti-Flag antibodies, followed by WB with anti-myc and LNK antibodies. Total cell lysates (TCLs) were also blotted as indicated. (B) 32D cells were infected with retroviruses encoding either WT or mutant Lnk. Cell growth inhibition was detected as described in Figure 3A. Representative results of 2 independent experiments are shown.

depletion of individual 14-3-3 isoforms $(\beta, \zeta$, or $\varepsilon)$ did not affect HSPC reconstitution (Figure 9B).

Since it is likely that 14-3-3 play redundant roles in HSPCs, we screened and identified shRNAs that simultaneously target multiple 14-3-3 isoforms. WB analysis identified 2 shRNA clones that diminished the production of $14-3-3 \beta$, $\varepsilon$, and $\zeta$ (referred to throughout as pan 14-3-3) in Lin- BM cells (Figure 9C). FACS analysis carried out prior to transplantation showed similar infection rates among all 3 groups (Figure 9D, left, and Figure 9E, time 0). $\mathrm{GFP}^{+}$populations were plotted as a fraction of donor-derived cells (Figure 9E) as well as a fraction of total host peripheral blood (PB) (Figure 9F). We observed that 14-3-3 depletion markedly inhibited HSPC reconstitution. Importantly, the degree of 14-3-3 knockdown correlated with the extent of inhibition.

To determine the blood reconstitution in different lineages, the percentages of $\mathrm{GFP}^{+}$cells in the myeloid and lymphoid compartments were monitored by FACS using lineage-specific markers. 14-3-3 depletion inhibited reconstitution of all examined lineages (Supplemental Figure 5). Of note, this was not due to a defect of HSPCs homing to the BM (Supplemental Figure 6). We further analyzed HSPC reconstitution in the host BM 16 weeks after transplant. Specifically, BM cells were analyzed for $\mathrm{GFP}^{+}$percentage in HSPCs using $\mathrm{Lin}-\mathrm{Sca}-1^{+} \mathrm{Kit}^{+}$(LSK) and SLAM markers (CD150+CD48-LSK) for long-term HSCs (ref. 38 and Supplemental Figure 7). This revealed that 14-3-3 depletion inhibited HSPC reconstitution in the BM (Supplemental Figure 7), suggesting for what we believe to be the first time that $14-3-3$ proteins play important and positive roles in HSC functions.

14-3-3 regulates HSPC functions in part through the LNK/JAK2/STAT5 pathway. To explore the signaling mechanisms by which 14-3-3 proteins affect HSPCs, we studied the effects of 14-3-3 depletion under conditions of LNK deficiency. Lin- BM cells from WT and $L n k^{-/-}$ mice were infected with lentiviruses expressing shRNA to Luc or pan 14-3-3 and transplanted into irradiated hosts (Figure 10, A and B). shRNA 14-3-3 no. 1 displayed the most effective knockdown of 14-3-3 (Figure 9C) and severely impaired BM reconstitution in both WT and Lnk-/ cells (Figure 10A), indicating that 14-3-3 proteins have multiple functions beyond interaction with LNK. Importantly, LNK deficiency mitigated the detrimental effects of shRNA14-3-3 no. 2 depletion (Figure 10B), which had an intermediate effect on 14-3-3 levels (Figure 9C), indicating that LNK contributes to functions of 14-3-3 proteins in HSPCs. Furthermore, 14-3-3 depletion dampened cytokine-induced STAT5 activation in HSPCs (Figure 10C). These results provide additional evidence supporting a positive role for 14-3-3 in promoting JAK/STAT signaling via LNK inhibition. 
A
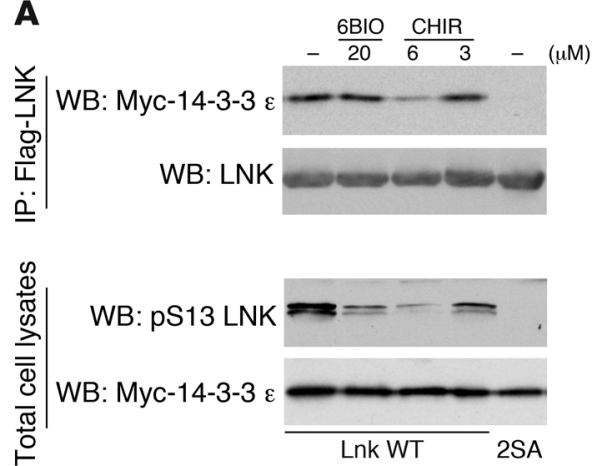

B

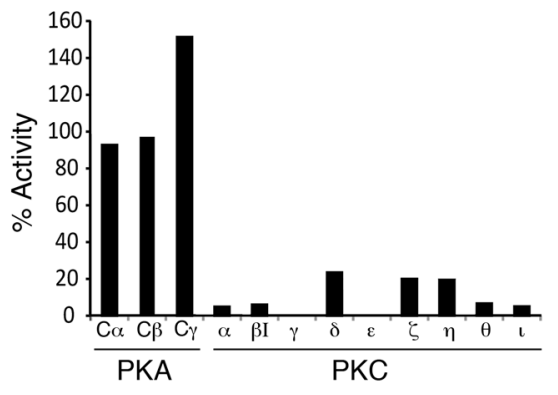

C

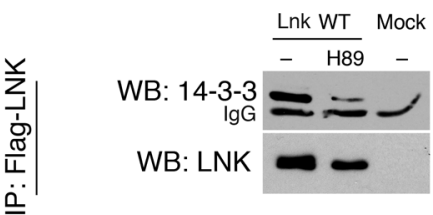

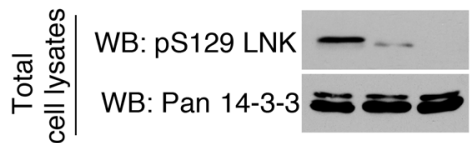

Figure 7

GSK3 and PKA phosphorylate LNK S13 and S129 sites, respectively. (A) 293T cells were cotransfected with myc-14-3-3 and Flag-Lnk WT or 2SA and treated with GSK3 inhibitors 2 hours prior to cell lysis. Lysates were either precipitated with anti-Flag antibodies followed by WB or directly subjected to WB using indicated antibodies. (B) In vitro kinase assays with various purified forms of PKA and PKC and LNK S129 peptides as substrates. Relative activities to known PKA and PKC substrates are shown. (C) 293T cells coexpressing 14-3-3 and Lnk were treated with PKA inhibitors $(20 \mu \mathrm{m}) 4$ hours prior cell lysis. Biochemical studies were performed as described in $\mathbf{A}$.

\section{Discussion}

LNK negatively regulates normal HSC expansion and self-renewal by restricting the TPO/MPL/JAK2 signaling pathway $(7,8,10)$. The work presented here provides insights into LNK function by demonstrating an interaction with 14-3-3 family proteins that we believe to be novel. Several lines of evidence support the importance of this interaction in regulating cytokine signaling and cell growth. First, mutations of the serine residues of LNK that mediate 14-3-3 binding lead to increased LNK activity, as reflected in augmented growth inhibitory activity and reduced JAK2 signaling. Second, restoration of 14-3-3 binding to mutant LNK restores normal LNK function. Third, depletion of 14-3-3 in hematopoietic HSPCs impairs their ability to repopulate lethally irradiated mice, a phenotype consistent with increased LNK activity (39). Fourth, LNK deficiency partially compensates for the loss of 14-3-3, further demonstrating the physiological importance of 14-3-3 as a key regulator of LNK in promoting HSPC functions. Fifth, 14-3-3 depletion dampens cytokine-induced STAT5 activation in HSPCs, thereby providing additional evidence supporting a positive role for 14-3-3 in promoting JAK/STAT signaling via LNK inhibition. Last, and importantly, mechanistic experiments revealed that 14-3-3 directly impairs LNK binding to JAK2 by subcellular sequestration, which agrees with the growth promoting effects of 14-3-3. Thus, our data reveal previously unappreciated serine phosphorylation events in LNK-dependent hematopoietic function and regulation of JAK2 signaling in hematopoiesis.

LNK is regulated by serine phosphorylation. Previous work established that LNK phosphorylation on tyrosine Y536 is important for LNK to execute its growth inhibitory function $(3,4)$, although the underlying mechanism was not defined. The present study in hematopoietic cell lines shows that LNK activity can be inhibited via serine phosphorylation, which leads to 14-3-3 binding. This in turn releases JAK2 from the constraints of LNK, thereby enhancing JAK2 signaling and cell proliferation. JAK2 does not affect serine phosphorylation of LNK, indicating that distinct pathways control serine and tyrosine phosphorylation events. Thus, LNK emerges as a dynamic signaling platform that is subjected to tightly balanced positive and negative regulatory modifications, allowing the integration of multiple signaling pathways.
It is important to note that basal levels of serine phosphorylation in LNK are notable, and TPO moderately increases these. What are the upstream kinases and extracellular signals beside TPO that regulate LNK serine phosphorylation? Our pharmacological inhibitors and in vitro kinase studies suggest S13 and S129 as potential substrates for GSK3 and PKA, respectively. Further work will be required to genetically establish LNK as a physiologically important substrate of these kinases, due to the limitations of pharmacological inhibitors. Of note, we recently reported that GSK3 indeed plays a pivotal role in HSC homeostasis and selfrenewal (35). A recent report in zebrafish suggest that prostaglandin E2 activates PKA in stem cells $(40,41)$. This illustrates that defining mechanisms by which LNK coordinates distinct signaling pathways not only enhances our knowledge of the cytokine signaling circuitry in HSCs but might lead to new pharmacological approaches by which HSC production can be improved, for instance through modulation of GSK3 or PKA.

14-3-3 proteins in HSPCs. 14-3-3 proteins have multiple client proteins and cellular functions (23), but their roles in HSPC expansion and hematopoietic development have been poorly explored. Our data provide mechanistic insight and for the first time to our knowledge reveal a biologic function of 14-3-3 proteins in primary HSPCs. In light of the difficulties in generating conditional knockout mice for all 7 14-3-3 isoforms, we used shRNAs that target multiple 14-3-3 isoforms expressed in BM cells. While knockdown of individual 14-3-3 isoforms did not affect HSPC reconstitution in BMT models, pan-14-3-3 knockdown markedly reduced the long-term reconstitution of HSPCs. Importantly, the extent to which blood reconstitution was attenuated correlates with the knockdown efficiencies. Therefore, our data establish that multiple forms of 14-3-3 play overlapping roles in HSPC development.

Multiple mechanisms can be invoked to explain these results, including effects of 14-3-3 on engraftment, proliferation, differentiation, and self-renewal of HSPCs. However, based on our findings that 14-3-3 knockdown did not affect HSPC homing and 14-3-3 acted directly on LNK to modulate JAK2 activity in hematopoietic cell lines, we favor a role in self-renewal (8). 14-3-3 proteins most likely have multiple targets in HSCs in addition to LNK. Furthermore, LNK deficiency mitigates the effects of 14-3-3 
A

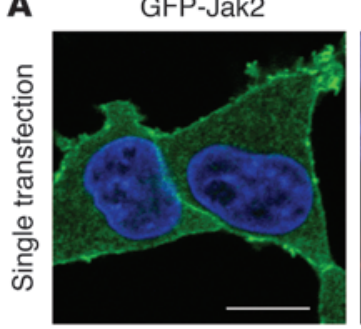

$\alpha G F P$
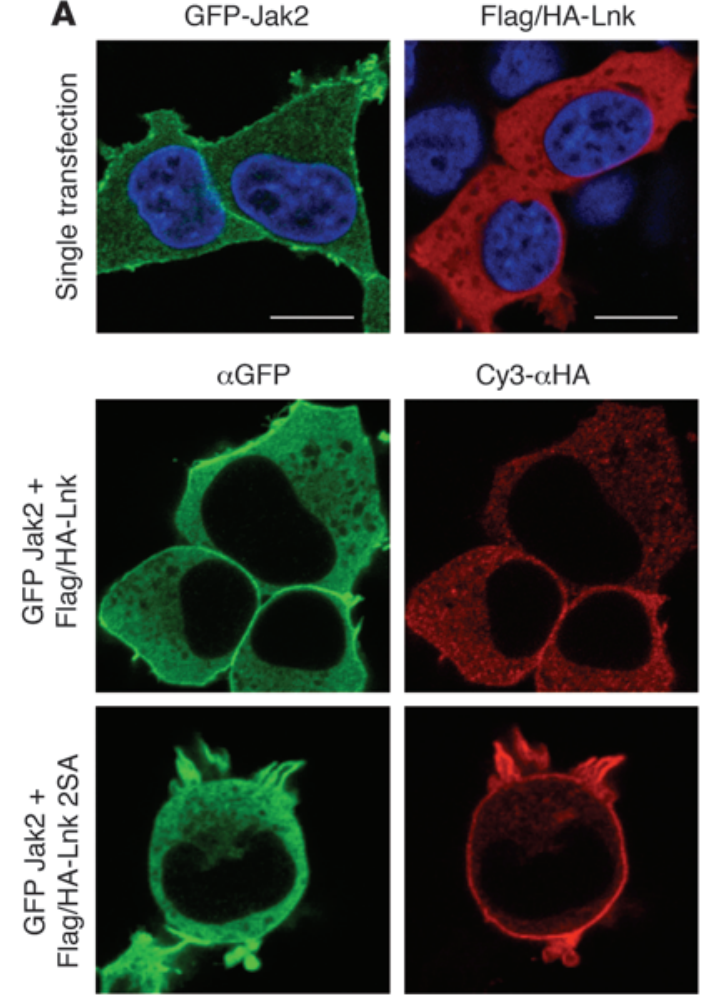

Cy3- $\alpha \mathrm{HA}$
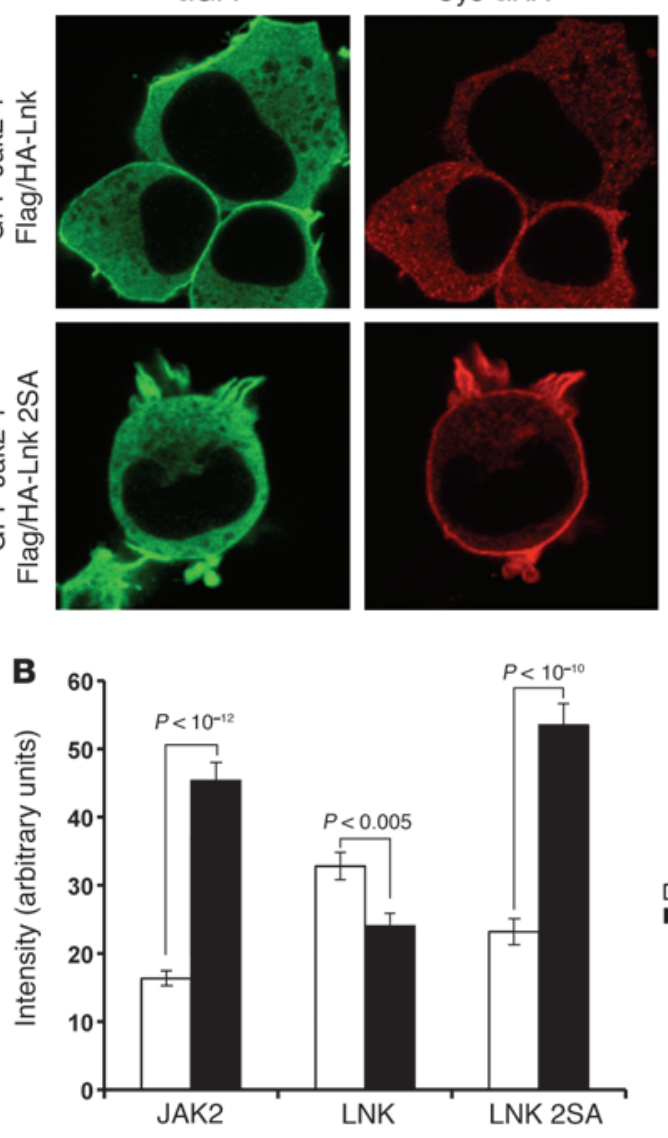

Flag/HA-Lnk 2SA

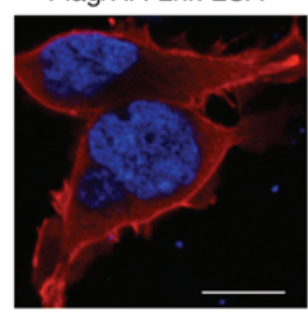

Merge + DAPI
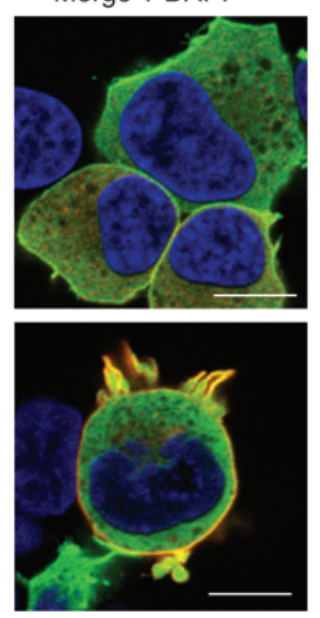

\section{Figure 8}

14-3-3 restrains LNK in the cytoplasm to prevent its association with the plasma membrane-proximal JAK2. (A) 293T cells were transfected with pEGFP-Jak2, pOZ-Lnk (Flag/HA-Lnk), or Lnk 2SA, either singly or in combinations. Two days later, cells were stained with anti-GFP and anti-HA antibodies, followed by Alexa Fluor 488 and Cy3 secondary antibodies, respectively. Confocal microscopy was performed, and representative images of 3 independent experiments are shown. Scale bars: $10 \mu \mathrm{m}$. (B) Intensities (average \pm SEM) of the cytoplasmic and plasma membrane signals. depletion in HSPC reconstitution assays, and, more importantly, 14-3-3 depletion dampens STAT5 activation. It suggests that 14-3-3 regulates HSPCs and hematopoiesis through both LNKdependent and -independent pathways, and inhibiting LNK/JAK2 pathway is one important mechanism by which $14-3-3$ proteins regulate HSPC function in vivo.

Mechanisms by which 14-3-3 interferes with the LNK-JAK2 interaction. Our data indicate that 14-3-3 binding to pS13 and pS129 of LNK directly inhibited LNK association with JAK2 in cell lines. It is important to note that 14-3-3 and JAK2 bind to distinct regions in LNK (JAK2 binds to the LNK SH2 domain at the C terminus) (Supplemental Figure 8). Therefore, it is unlikely that there is a direct competition in LNK association between 14-3-3 and JAK2. Our confocal microscopy data suggest that 14-3-3 binding sequesters LNK away from JAK2, which is associated with the plasma membrane, thereby limiting their interactions. Of note, we cannot exclude the possibility that other binding proteins through LNK S13 and S129 could affect LNK localization. There are precedents for 14-3-3 in relocating its client proteins (42). For example, in response to AKT phosphorylation, FOXO binds to 14-3-3, resulting in sequestration of FOXO in the cytoplasm (42). Moreover, we show that 14-3-3 modulates JAK/STAT signaling in primary HSPCs. Therefore, our studies reveal mechanisms by which 14-3-3 impinges upon cytokine signaling in hematopoiesis.

LNK dysfunction in MPNs. LNK deficiency predisposes mice toward MPNs caused by oncogenic JAK2. In addition, LNK deficiency by itself leads to MPNs in aged animals at least in part via augmented JAK2 signaling $(12,13)$. Importantly, missense and deletion mutations in LNK are found in patients with MPN as well as blast-phase MPNs or secondary AMLs $(15,16)$. Human CD34+ progenitors bearing LNK mutations showed aberrant activation of STAT5 and Stat3 (16). These data strongly implicate an important role for LNK in restraining JAK/Stat signaling and MPN development.

While overall genetic disruption of LNK in MPNs is rare (3\%-6\%) $(17,43)$, structural alterations of Lnk may not be the only disruptions that can promote MPN development. Our data strongly sug- 
A

shRNA sh14-3-3 $\beta$

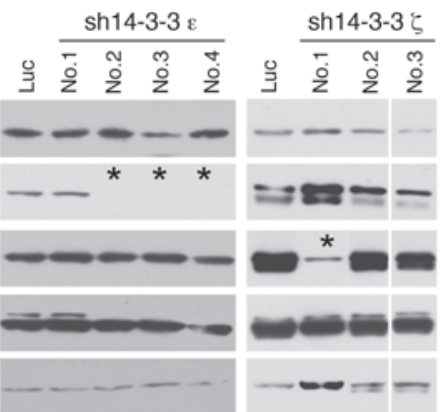

C

sh Pan

14-3-3

$14-3-3 \varepsilon=0$

$14-3-3 \xi$

Pan 14-3-3

Actin

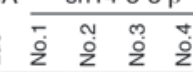

14-3-3

$--$

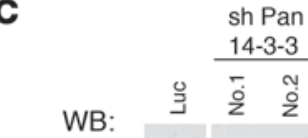

14-3-3 $\beta$

$14-3-3 \varepsilon=$

$14-3-3 \xi$

Pan 14-3-3

Actin $-\ldots$

D
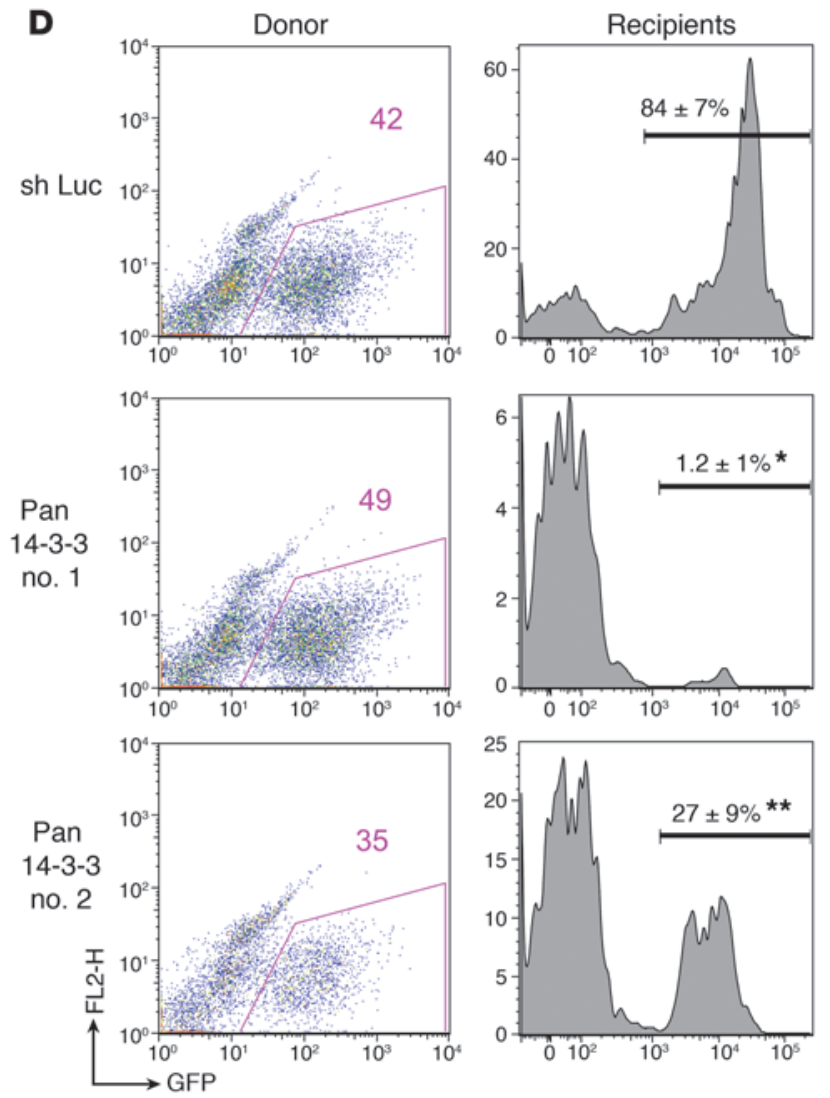

B
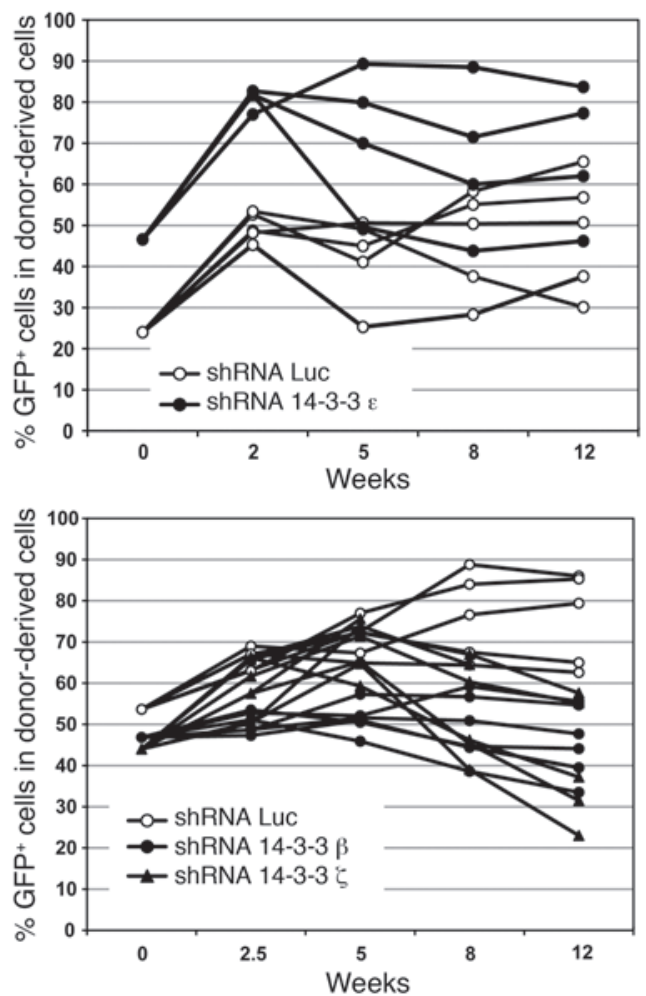

E
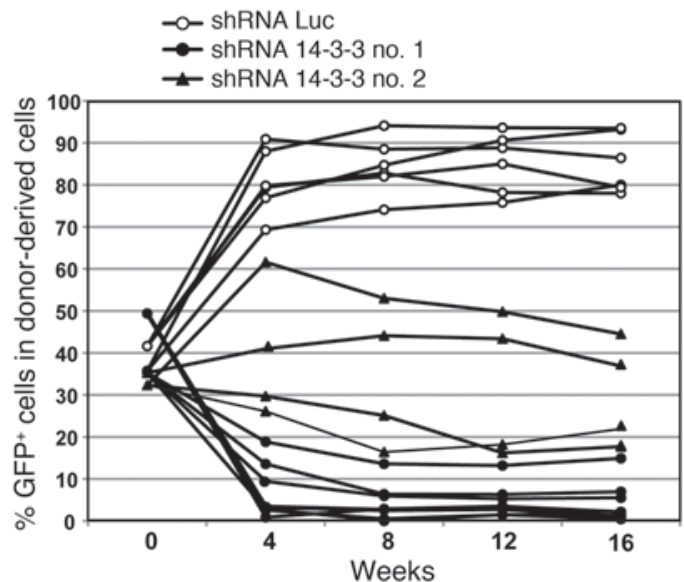

$\mathbf{F}$

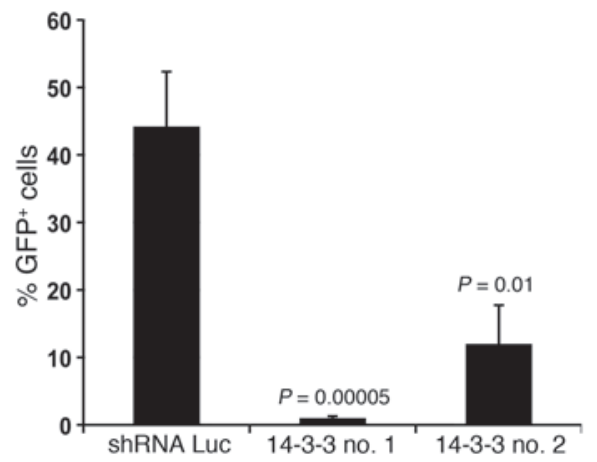




\section{Figure 9}

HSPC reconstitution in mice requires 14-3-3 proteins. (A) Lin- BM cells were infected with pLKO-shRNA lentiviruses to either Luc or 14-3-3 $\varepsilon$, $\beta$, or $\xi$. Knockdown efficiencies were examined by WB. Asterisks indicate clones with high knockdown efficiencies. All the lanes were run on the same gel but were noncontiguous. No., indicates clone number. (B) Infected Lin- BM cells were transplanted into lethally irradiated recipient mice. The percentages of $\mathrm{GFP}^{+}$reconstitutions in the PB of host animals 4, 8, and 12 weeks after BMT are shown. Time 0 indicates time of transplant. Each line represents an individual transplanted animal. (C-F) Pan 14-3-3 knockdown inhibits HSPC reconstitution. (C) Lin- BM cells were infected with pLKO-shRNA lentiviruses to either Luc or 2 pan 14-3-3 clones (no. 1 or no. 2). Knockdown efficiencies were examined by WB. All the lanes were run on the same gel but were noncontiguous. (D) Lin- BM cells expressing shRNAs were transplanted. FACS plots of the percentages of GFP+ in donor BM cells before transplant and host PB 16 weeks after transplant are shown. Results are pooled from 2 independent experiments. ${ }^{*} P<0.0001$, ${ }^{*} P<0.01$, compared with Luc control. (E) The percentage of GFP+ reconstitutions in donor-derived PB of host animals at indicated times is shown. (F) The percentage of GFP+reconstitutions in total PB of host animals at 12 weeks is shown $(n=6-10)$. Average \pm SEM.

gest that dysregulation of posttranslational modifications within the Lnk/JAK2 signaling pathway could also influence initiation and progression of MPNs. The findings in this report showing that LNK is negatively regulated by serine phosphorylation warrant further studies examining LNK phosphorylation status in human MPNs. By extension, 14-3-3 proteins might be structurally or functionally altered in human neoplasms. Indeed, 14-3-3 proteins are overexpressed in breast and squamous cell carcinomas, and 14-3-3 overexpression levels in breast cancers correlate with metastatic recurrence and poor survival (44-46). Furthermore, 14-3-3 disruption by a competitive antagonist induced significant apoptosis in Bcr/Abl-transformed hematopoietic cells (47). Taken together, further studies are warranted on the role of 14-3-3 functions in hematopoietic malignancies.

We also observed that LNK interacts with JAK1 in addition to JAK2 (our unpublished observations). Therefore, it is possible that acute lymphoblastic leukemia-associated (ALL-associated) JAK mutants might also be under the control of the LNK-14-3-3 system. Importantly, LNK mutations have been found in T-ALL (48). Therefore, our findings warrant future studies to examine LNK mutations and 14-3-3 overexpression in additional hematologic malignancies. Furthermore, LNK and 14-3-3 stand as potential targets to modulate relevant signaling pathways in these diseases.

\section{Figure 10}

14-3-3s regulate HSPC reconstitution in mice partially through the LNK/JAK2/Stat5 pathway. (A and B) Lin- BM cells from WT and Lnk-1mice were infected with lentiviruses expressing shRNA to either Luc or (A) pan 14-3-3 no. 1 or (B) no. 2 and subsequently transplanted. The percentages of $\mathrm{GFP}^{+}$cells before transplant and in host PB after transplant are shown $(n=5-7)$. Average \pm SEM. (C) Lin- BM cells were infected with pLKO-Luc or pan 14-3-3 no. 2, and subsequently purified GFP+Lin-Sca1+ progenitors were stimulated with or without IL-3 for 10 minutes and subjected to phospho-flow analysis using anti-pStat5 antibodies. Histograms of unstimulated and stimulated cells are overlaid and shown. shLuc-infected cells are labeled in red, while the sh14-3-3 cells are labeled in blue. Dashed lines indicate unstimulated cells, while solid lines indicate stimulated cells. Shaded areas represent secondary antibody-only controls.

\section{Methods}

Mice and cell lines. Lnk ${ }^{-/-}$mice were provided by Tony Pawson and Laura Velazquez (Samuel Lunenfeld Research Institute, Toronto, Ontario, Canada) (6) and backcrossed onto the C57/BL6 background for 9 generations. IL-3-dependent 32D clone 3 cells were purchased from ATCC. 32D cells were maintained in RPMI containing $10 \%$ heat-inactivated fetal calf serum (Hyclone, Thermo Fisher Scientific) and 10\% WEHI-3B cell supernatant as a source of IL-3 (WEHI media).

Constructs. Retroviral MIG-Lnk (MSCV-Lnk-ires-GFP) and pOZ-Lnk (MSCV-Flag/HA-Lnk-ires-IL-2R) constructs were generated as described previously $(4,12)$. The pOZ-Lnk 2SA mutant was generated through sitedirected mutagenesis using a QuikChange Kit and confirmed by sequencing (Stratagene). pMICD4-myc-tagged 14-3-3 was cloned by Polymerase Chain Reaction (PCR) 14-3-3 cDNA sequence with XhoI and EcoRI sites at the $5^{\prime}$ and $3^{\prime}$ oligos, respectively, and subsequently ligated to PMICD4 (MSCV-ires-CD4 vector containing 6x myc tag at the $\mathrm{N}$ terminus) (3) linearized with XhoI and EcoRI. Lentiviral pLKO.1-puro shRNA to Luc or 14-3-3 were purchased from Open Biosystems. The sequence encoding puromycin gene was excised with BamH1 and Kpn1 enzymes and replaced with the GFP cDNA sequence to generate pLKO-GFP constructs.

Viral packaging and infection. Retroviral constructs were cotransfected with pCL-Eco (49) into 293T Phoenix packaging cells (Garry Nolan, Stanford University, Stanford, California, USA). For lentiviral packaging, lentiviral transfer constructs were cotransfected with pMDLg/p, pRSV-rev, and pMDG.2-env packaging constructs according to standard protocols (50).

The resulting supernatant was collected 48 hours later. 32D cells or Lin$\mathrm{BM}$ cells were spin-infected with the desired viral supernatant containing $10 \mu \mathrm{g} / \mathrm{ml}$ polybrene (Sigma-Aldrich) at $640 \mathrm{~g}$ for 1 hour at $30^{\circ} \mathrm{C}$.

Phospho-flow cytometric analysis. 32D cells were starved for 2 hours, and either left unstimulated or stimulated with $20 \mathrm{ng} / \mathrm{ml}$ TPO for 10 minutes. After stimulation, cells were fixed with $4 \%$ freshly prepared paraformaldehyde solution and permeabilized with ice-cold methanol (51). Cells were then stained with anti-IL-2R antibodies (Upstate Biotechnology), together with anti-pY1007/8 JAK2 antibodies (no. 3771) or p-STAT5 (no. 9351 and no. 4322), pAKT (no. 4058), and pERK (no. 9101) all from Cell Signaling Technology at 1:100 dilution, followed by anti-rabbit IgG secondary antibodies conjugated to Alexa Fluor 488 (Molecular Probes; $1: 400)$ and anti-mouse secondary antibodies conjugated to APC (BD Biosciences; 1:400), and analyzed on a FACSCalibur.

$\mathrm{Lin}^{-} \mathrm{BM}$ infection and transplantation. Mice were directly sacrificed or were treated with 5-fluorouracil to enrich for HSPCs prior to BM harvest. Lin cells were isolated from the BM using a panel of Lin antibodies followed by Dynabeads depletion of $\mathrm{Lin}^{+}$cells, according to the manufacturer's protocol $(8,12)$. Then the cells were cultured in liquid media containing multiple cytokines: SFEM (StemCell Technologies Inc.) containing 10\% FBS, 50 $\mu \mathrm{M} \beta$-mercaptoethanol, $100 \mathrm{ng} / \mathrm{ml}$ stem cell factor (SCF), $10 \mathrm{ng} / \mathrm{ml} \mathrm{IL-3,}$ $10 \mathrm{ng} / \mathrm{ml} \mathrm{IL-6}$, and $10 \mathrm{ng} / \mathrm{ml}$ TPO (PeproTech). Lin- BM progenitor cells were infected with pLKO lentiviruses. Subsequently, infected cells were selected in $4 \mu \mathrm{g} / \mathrm{ml}$ puromycin for 2 days in culture and then plated in semisolid methylcellulose media. Cells were collected and processed for WB analysis for 14-3-3 protein expression.

After determination of 14-3-3 knockdown efficiencies, we replaced the sequence for puromycin selection marker with the GFP sequence to generate pLKO-GFP constructs. Lin ${ }^{-}$cells from C57/B6 CD45.2 mice were isolated and infected with pLKO-GFP lentiviruses expressing shRNAs and then transplanted into lethally irradiated B6.SJL CD45.1 recipients. The infection rates were determined by FACS analysis for GFP expression on the day of transplantation. After transplantation, chimerisms in the PB of recipient mice were analyzed for GFP and CD45.2 levels by flow cytometry. Lineage reconstitutions were determined with different lineage markers: with Gr-1 and Mac-1 for myeloid lineage and CD3e and CD19 for T and B cell lineages, respectively. All antibodies were obtained from eBiosciences, and flow cytometry analysis was carried out as previously described (52).

BrdU incorporation assay. Lnk $^{-/}$Lin $^{-} \mathrm{BM}$ cells were spin-infected with pOZLnk or Lnk 2SA retroviruses and cultured as described above. Twenty-four hours later, $10 \mu \mathrm{M} \mathrm{BrdU}$ was added to the cell culture for 45 minutes. Infected populations were measured by FACS analysis using APC-conjugated antiIL-2R antibodies. At the same time, BrdU incorporations were determined using FITC-conjugated anti-BrdU antibodies and 7 '-aminoactinomycin D ( $7^{\prime}$-AAD), according to manufacturer's instruction (BD Biosciences).
Confocal immunofluorescence microscopy. The pEGFP-C1-Jak2 construct was provided by Lily Jun-Shen Huang (UT Southwestern Medical Center, Dallas, Texas, USA). Two days after transfection, 293T cells were fixed with paraformaldehyde, permeabilized with saponin, and stained with anti-GFP (Invitrogen) and anti-HA antibodies (HA11.1, Covance), followed by Alexa Fluor 488-conjugated anti-rabbit IgG and Cy3-conjugated anti-mouse IgG secondary antibodies (Molecular Probes). Confocal images were acquired under a Zeiss LM710 microscope. Confocal microscope images were quantified by ImageJ. Briefly, a line was drawn on the cytoplasmic or membrane portion of each cell, and intensities along the line were obtained using the Analyze-Plot profile function. Intensities were then averaged from 20 to 30 cells from 3 independent experiments.

IP and WB analysis. Cells were lysed in buffer containing $0.5 \%$ NP-40, phosphatase, and protease inhibitors as described in ref. 4 , with the addition of $\beta$-glycerolphosphate and sodium pyrophosphate to prevent serine/ threonine phosphatase activities. IP was preformed with anti-Flag beads (Sigma-Aldrich), or myc (9E10, Covance) antibodies. WB was performed with anti-phosphotyrosine $(0.5 \mu \mathrm{g} / \mathrm{ml} ; 4 \mathrm{G} 10$, Upstate); myc (9B11, Cell Signaling Technology); and 14-3-3 $\beta$ (C-20), 14-3-3 ع (5A5), 14-3-3 $\zeta$ (C-16), $14-3-3 \eta$ (K-12), pan 14-3-3 antibodies (H-8 and K-19), and anti-LNK antibodies (all at 1:1,000; all from Santa Cruz Biotechnology).

Statistics. Two-tailed Student's $t$ tests were used. A $P$ value of less than 0.05 was considered significant.

Study approval. The protocol for this work was approved by the Institutional Animal Care and Use Committee of the Children's Hospital of Philadelphia.

\section{Acknowledgments}

W. Tong was supported by NIH grants R01HL095675, R01HL110806, and R21HL102688. W. Tong received a New Investigator Award from the Myeloproliferative Neoplasm Research Foundation and Gabrielle's Angel Foundation for Cancer Research. A. Bersenev and Y. Song were supported by NIH training grants T32HL007971 and T32DK007780, respectively. J. Jiang and $\mathrm{K}$. Rozenova were supported by postdoctoral fellowships from the American Heart Association. We are grateful to Gerd Blobel for critical review of the manuscript.

Received for publication June 28, 2011, and accepted in revised form March 9, 2012.

Address correspondence to: Wei Tong, Abramson Bldg., Suite 316A, Children's Hospital of Philadelphia, 3615 Civic Center Boulevard, Philadelphia, Pennsylvania 19104-4318, USA. Phone: 267.426.0930; Fax: 267.426.5476; E-mail: tongw@email.chop.edu.
1. Kaushansky K. Thrombopoietin: a tool for understanding thrombopoiesis. J Thromb Haemost. 2003; 1(7):1587-1592.

2. Takaki S, et al. Control of B cell production by the adaptor protein lnk. Definition of a conserved family of signal-modulating proteins. Immunity. 2000;13(5):599-609.

3. Tong W, Zhang J, Lodish HF. Lnk inhibits erythropoiesis and Epo-dependent JAK2 activation and downstream signaling pathways. Blood. 2005; 105(12):4604-4612.

4. Tong W, Lodish HF. Lnk inhibits Tpo-mpl signaling and Tpo-mediated megakaryocytopoiesis. J Exp Med. 2004;200(5):569-580.

5. Takaki S, Morita H, Tezuka Y, Takatsu K. Enhanced hematopoiesis by hematopoietic progenitor cells lacking intracellular adaptor protein, Lnk. J Exp Med. 2002;195(2):151-160.
6. Velazquez L, et al. Cytokine signaling and hematopoietic homeostasis are disrupted in Lnk-deficient mice. J Exp Med. 2002;195(12):1599-1611.

7. Seita J, et al. Lnk negatively regulates self-renewal of hematopoietic stem cells by modifying thrombopoietin-mediated signal transduction. Proc Natl Acad Sci U S A. 2007;104(7):2349-2354.

8. Bersenev A, Wu C, Balcerek J, Tong W. Lnk controls mouse hematopoietic stem cell self-renewal and quiescence through direct interactions with JAK2. J Clin Invest. 2008;118(8):2832-2844.

9. Ema H, et al. Quantification of self-renewal capacity in single hematopoietic stem cells from normal and Lnk-deficient mice. Dev Cell. 2005;8(6):907-914

10. Buza-Vidas $\mathrm{N}$, et al. Cytokines regulate postnatal hematopoietic stem cell expansion: opposing roles of thrombopoietin and LNK. Genes Dev. 2006; 20(15):2018-2023.
11. Tefferi A, Gilliland DG. Oncogenes in myeloproliferative disorders. Cell Cycle. 2007;6(5):550-566.

12. Bersenev A, et al. Lnk constrains myeloproliferative diseases in mice. J Clin Invest. 2010;120(6):2058-2069.

13. Gery S, Cao Q, Gueller S, Xing H, Tefferi A, Koeffler HP. Lnk inhibits myeloproliferative disorderassociated JAK2 mutant, JAK2V617F. J Lenkoc Biol. 2009;85(6):957-965

14. Baran-Marszak F, et al. Expression level and differential JAK2-V617F-binding of the adaptor protein Lnk regulates JAK2-mediated signals in myeloproliferative neoplasms. Blood. 2010;116(26):5961-5971.

15. Lasho TL, Pardanani A, Tefferi A. LNK mutations in JAK2 mutation-negative erythrocytosis. $N$ Engl J Med. 2010;363(12):1189-1190.

16. Oh ST, et al. Novel mutations in the inhibitory adaptor protein LNK drive JAK-STAT signaling in patients with myeloproliferative neoplasms. Blood. 
2010;116(6):988-992.

17. Pardanani A, Lasho T, Finke C, Oh ST, Gotlib J, Tefferi A. LNK mutation studies in blast-phase myeloproliferative neoplasms, and in chronic-phase disease with TET2, IDH, JAK2, or MPL mutations. Lenkemia. 2010;24(10):1713-1718.

18. Morrison DK. The 14-3-3 proteins: integrators of diverse signaling cues that impact cell fate and cancer development. Trends Cell Biol. 2009;19(1):16-23.

19. Tzivion G, Gupta VS, Kaplun L, Balan V. 14-3-3 proteins as potential oncogenes. Semin Cancer Biol. 2006;16(3):203-213.

20. Muslin AJ, Tanner JW, Allen PM, Shaw AS. Interaction of 14-3-3 with signaling proteins is mediated by the recognition of phosphoserine. Cell. 1996; 84(6):889-897.

21. Yaffe MB, et al. The structural basis for 14-3-3: phosphopeptide binding specificity. Cell. 1997; 91(7):961-971.

22. Rittinger K, et al. Structural analysis of 14-3-3 phosphopeptide complexes identifies a dual role for the nuclear export signal of 14-3-3 in ligand binding. Mol Cell. 1999;4(2):153-166.

23. Bridges D, Moorhead GB. 14-3-3 proteins: a number of functions for a numbered protein. Sci STKE. 2005;2005(296):re10.

24. Toyo-oka K, et al. 14-3-3epsilon is important for neuronal migration by binding to NUDEL: a molecular explanation for Miller-Dieker syndrome. Nat Genet. 2003;34(3):274-285.

25. Lau JM, et al. The 14-3-3tau phosphoserine-binding protein is required for cardiomyocyte survival. Mol Cell Biol. 2007;27(4):1455-1466.

26. Li Q, Lu Q, Estepa G, Verma IM. Identification of 14-3-3sigma mutation causing cutaneous abnormality in repeated-epilation mutant mouse. Proc Natl Acad Sci U S A. 2005;102(44):15977-15982.

27. Steinacker $P$, et al. Unchanged survival rates of 14-3-3gamma knockout mice after inoculation with pathological prion protein. Mol Cell Biol. 2005; 25(4):1339-1346

28. Barry EF, et al. 14-3-3:Shc scaffolds integrate phosphoserine and phosphotyrosine signaling to regulate phosphatidylinositol 3-kinase activation and cell survival. J Biol Chem. 2009;284(18):12080-12090.

29. Jin $\mathrm{HO}$, et al. Hypoxic condition- and high cell density-induced expression of Redd 1 is regulated by activation of hypoxia-inducible factor-1alpha and Sp1 through the phosphatidylinositol 3-kinase/Akt signaling pathway. Cell Signal. 2007;19(7):1393-1403.

30. $\mathrm{Yu} \mathrm{D}$, et al. miR-451 protects against erythroid oxidant stress by repressing 14-3-3zeta. Genes Dev. 2010;24(15):1620-1633.

31. Patrick DM, et al. Defective erythroid differentiation in miR-451 mutant mice mediated by 14-3-3zeta. Genes Dev. 2010;24(15):1614-1619.

32. Obenauer JC, Cantley LC, Yaffe MB. Scansite 2.0: Proteome-wide prediction of cell signaling interactions using short sequence motifs. Nucleic Acids Res. 2003;31(13):3635-3641

33. Wang $B$, et al. Isolation of high-affinity peptide antagonists of 14-3-3 proteins by phage display. Biochemistry. 1999;38(38):12499-12504.

34. Ramm G, Larance M, Guilhaus M, James DE. A role for 14-3-3 in insulin-stimulated GLUT4 translocation through its interaction with the RabGAP AS160. J Biol Chem. 2006;281(39):29174-29180.

35 . Huang J, et al. Pivotal role for glycogen synthase kinase-3 in hematopoietic stem cell homeostasis in mice. J Clin Invest. 2009;119(12):3519-3529.

36. Gery S, Gueller S, Chumakova K, Kawamata N, Liu L, Koeffler HP. Adaptor protein Lnk, negatively regulates the mutant MPL, MPLW515L, associated with myeloproliferative disorders. Blood. 2007;110(9):3360-3364.

37. Krivtsov AV, et al. Transformation from committed progenitor to leukaemia stem cell initiated by MLL-AF9. Nature. 2006;442(7104):818-822.

38. Kiel MJ, Yilmaz OH, Iwashita T, Terhorst C, Morrison SJ. SLAM family receptors distinguish hematopoietic stem and progenitor cells and reveal endothelial niches for stem cells. Cell. 2005;121(7):1109-1121.

39. Takizawa $\mathrm{H}$, et al. Enhanced engraftment of hematopoietic stem/progenitor cells by the transient inhibition of an adaptor protein, Lnk. Blood. 2006;107(7):2968-2975.

40. North TE, et al. Prostaglandin E2 regulates vertebrate haematopoietic stem cell homeostasis. Nature.
2007;447(7147):1007-1011.

41. Goessling W, et al. Genetic interaction of PGE2 and Wnt signaling regulates developmental specification of stem cells and regeneration. Cell. 2009; 136(6):1136-1147.

42. Accili D, Arden KC. FoxOs at the crossroads of cellular metabolism, differentiation, and transformation. Cell. 2004;117(4):421-426.

43. Abdel-Wahab O. Genetics of the myeloproliferative neoplasms. Curr Opin Hematol. 2011;18(2):117-123.

44. Wang Z, Nesland JM, Suo Z, Trope CG, Holm R. The prognostic value of 14-3-3 isoforms in vulvar squamous cell carcinoma cases: 14-3-3beta and epsilon are independent prognostic factors for these tumors. PLoS One. 2011;6(9):e24843.

45. Lu J, et al. 14-3-3zeta Cooperates with ErbB2 to promote ductal carcinoma in situ progression to invasive breast cancer by inducing epithelial-mesenchymal transition. Cancer Cell. 2009;16(3):195-207.

46. Neal CL, et al. 14-3-3zeta overexpression defines high risk for breast cancer recurrence and promotes cancer cell survival. Cancer Res. 2009;69(8):3425-3432.

47. Dong S, Kang S, Lonial S, Khoury HJ, Viallet J, Chen J. Targeting 14-3-3 sensitizes native and mutant BCRABL to inhibition with U0126, rapamycin and Bcl-2 inhibitor GX15-070. Lenkemia. 2008;22(3):572-577.

48. Zhang J, et al. The genetic basis of early T-cell precursor acute lymphoblastic leukaemia. Nature. 2012;481(7380):157-163.

49. Naviaux RK, Costanzi E, Haas M, Verma IM. The PCL vector system: rapid production of helper-free, high-titer, recombinant retroviruses. J Virol. 1996; 70(8):5701-5705.

50. Dull T, et al. A third-generation lentivirus vector with a conditional packaging system. J Virol. 1998; 72(11):8463-8471.

51. Krutzik PO, Nolan GP. Intracellular phospho-protein staining techniques for flow cytometry: monitoring single cell signaling events. Cytometry A. 2003; 55(2):61-70.

52. Tong W, Ibarra YM, Lodish HF. Signals emanating from the membrane proximal region of the thrombopoietin receptor $(\mathrm{mpl}$ ) support hematopoietic stem cell self-renewal. Exp Hematol. 2007;35(9):1447-1455. 